\title{
3 A Multimethod Approach for Investigating Algal Toxicity of Platinum Nanoparticles
}

4

5 Sara N. Sørensen ${ }^{\dagger *}$, Christian Engelbrekt ${ }^{\ddagger}$, Hans-Christian H. Lützhøft ${ }^{\dagger}$, Javier Jiménez-Lamana ${ }^{\S}$, Jafar

6 Noori $^{\|}$, Fatima A. Alatraktchi ${ }^{\perp}$, Cristina G. Delgado ${ }^{\dagger}$, Vera I. Slaveykova ${ }^{\S}$ and Anders Baun ${ }^{\dagger}$

7

8 Corresponding author

$9 \quad$ Name: $\quad$ Sara Nørgaard Sørensen

10 Address: Department of Environmental Engineering, Technical University of Denmark Miljøvej, Building 113, DK-2800 Kongens Lyngby, Denmark

12 Phone: $\quad+4545251688$ or +4528556482

$13 \quad$ Fax: $\quad+4545932850$

$14 \quad$ E-mail: $\quad \underline{\text { sans@env.dtu.dk }}$

15

16 This supporting information contains:

1716 Pages

$18 \quad 2$ Tables

199 Figures 
22 S1 Materials and methods - additional details including references

Page S3

23 S2 Settings and results for AsFIFFF measurements

Page S7

24 S3 Experimental setup for shading experiments

Page S8

25 S4 Flow cytometry gating strategies

Page S9

26 S5 Sedimentation of PtNPs in algal media

Page S12

27 S6 Abiotic ROS generation by $\mathrm{PtNPs}$ and $\mathrm{PtC}_{14}$ in algal media

Page S13

28

S7 Potential PSII photochemical efficiency in algae

Page S14

29 S8 Membrane damage in algal cells

Page S15

30

S9 Atomic force microscopy images of algal cells

Page S16 
Size distribution measured by AsFIFFF

34 Suspensions of $4 \mathrm{mg} \mathrm{Pt} / \mathrm{L}$ were injected in the separation system and characterized immediately upon

preparation and after 1, 24 and $48 \mathrm{~h}$. A spacer of $350 \mu \mathrm{m}$ thickness, a $20 \mu \mathrm{L}$ loop and a regenerated cellulose (RC) membrane with a cut-off of $1 \mathrm{kDa}$ were used in all the experiments. $0.01 \%$ sodium dodecyl sulfate (SDS) in $1 \mathrm{mM} \mathrm{NH}_{4} \mathrm{NO}_{3}$ (Sigma-Aldrich) solution at $\mathrm{pH} 8$ was used as carrier. The optimal separation program found is detailed in Table S1. In order to characterize the sample, the system was first calibrated against polystyrene standards of known size (22, 58 and $97 \mathrm{~nm})$. The following linear relationship between the logarithm of the retention ratio $\mathrm{R}$ (defined as elution time corresponding to the void volume divided by the retention time for a given particle) and the logarithm of the diameter (d) in nanometers was experimentally found:

$$
\log (R)=0.4939-0.9539 \times \log (d) ; r=0.9958
$$

Fractograms obtained as a function of time for each sample were converted into size distributions according to equation (1).

\section{Abiotic ROS formation}

$\mathrm{H}_{2}$ DCF-DA was dissolved in ethanol $(1.3 \mathrm{mM})$ and deacetylated to $\mathrm{H}_{2}$ DCF by letting $1 \mathrm{~mL}$ react with 4 $\mathrm{mL} 0.01 \mathrm{M} \mathrm{NaOH}$ for $30 \mathrm{~min}$ in the dark. The mixture was added $20 \mathrm{~mL}$ sodium phosphate buffer (25

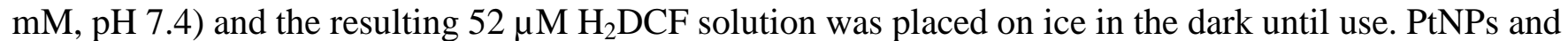
$\mathrm{PtCl}_{4}$ were added to TAP4 and ISO media in the concentration range 0.001-390 mg Pt/L. After $2 \mathrm{~h}$ and 48 $\mathrm{h}$ incubation in media, under the same conditions as for growth inhibition testing, $100 \mu \mathrm{L}$ of the PtNP suspensions was each mixed with $100 \mu \mathrm{L} \mathrm{H}_{2}$ DCF solution in the wells of a 96-well black microplate (three replicates of each concentration). After $1 \mathrm{~h}$ incubation in the dark, the fluorescence of DCF was 
60

61

62

63

64

65

\section{6}

measured (readings, $\mathrm{n}=3$ ) (excitation/emission at 485/527 nm) using a fluorescence plate reader (Biotek Synergy Mx plate reader). As positive control, $\mathrm{H}_{2} \mathrm{O}_{2}$ was diluted in ISO and TAP4 media in the concentration range $0.16-20 \%$ (w/w). The ROS level in samples was calculated in relative fluorescence units (RFUs) by dividing the fluorescence of samples (PtNPs in media incubated with $\mathrm{H}_{2} \mathrm{DCF}$ ) by fluorescence of background (media incubated with $\mathrm{H}_{2} \mathrm{DCF}$ ).

\section{Algal culturing}

P. subcapitata was obtained from the Norwegian Institute for Water Research, Oslo, Norway (NIVA) and cultivated in ISO 8692 medium $^{1}$ and the C. reinhardtii strain CPC11 was obtained from the Canadian Phycological Culture Center (CPCC, Department of Biology, University of Waterloo, Canada) and grown in four times diluted Tris-Acetate-Phosphate medium ${ }^{2}$. The two respective media are hereafter referred to as ISO and TAP4 media. For all toxicity testing, the algae were exposed in their respective cultivation medium and testing was conducted under the same incubation conditions as for the culture. For cellular toxicity, photosynthesis efficiency and body burden studies $250 \mathrm{~mL}$ Erlenmeyer flasks were fitted with permeable stoppers, containing $50 \mathrm{~mL}$ algal suspension and incubated (Infors, Bottmingen, Switzerland) at $20 \pm 2^{\circ} \mathrm{C}$ with continuous agitation $(100 \mathrm{rpm})$ and illumination from above $\left(110 \pm 10 \mu \mathrm{mol} / \mathrm{m}^{2} / \mathrm{s}\right)$. For growth and carbon assimilation inhibition tests $20 \mathrm{~mL}$ glass vials with perforated screw cap lids, containing $5 \mathrm{~mL}$ suspension were kept at $20 \pm 2^{\circ} \mathrm{C}$, continuous shaking (300 rpm) and illuminated from below by fluorescent tubes (30W/33; Philips, The Netherlands) at a light intensity of $100 \pm 20 \mu \mathrm{mol} / \mathrm{m}^{2} / \mathrm{s}$. The algal cultures were re-inoculated in fresh media every second to third day, to ensure an exponentially growing culture.

\section{Algal ${ }^{14} \mathrm{C}$-assimilation tests}


Preparation of test concentrations, control and replicates was similar to the growth rate inhibition tests, but the initial algal density was $10^{5}$ cells/mL. Immediately before incubation, $50 \mu \mathrm{L}$ of $\mathrm{NaH}^{14} \mathrm{CO}_{3}$ solution (specific activity: $20 \mu \mathrm{Ci} / \mathrm{mL}$; obtained from DHI, Hoersholm, Denmark) was added to all vials which were then closed with airtight screw caps. The tests were terminated after $2 \mathrm{~h}$ incubation by adding $0.2 \mathrm{~mL}$ $10 \% \mathrm{HCl}$ to each vial (yielding $\mathrm{pH}<2$ ). The vials were left open overnight in a fume hood and $10 \mathrm{~mL}$ scintillation liquid (Optiphase "Hisafe” 3, Perkin Elmer, Waltham, MA, USA) was added to each vial. After thorough mixing, they were left in the dark for $8 \mathrm{~h}$ and submitted to liquid scintillation counting (Hidex $300 \mathrm{SL}$ ). $\mathrm{H}^{14} \mathrm{CO}_{3}$ solution was also added to three replicates of medium only, as controls to confirm that all added ${ }^{14} \mathrm{C}$ that had not incorporated into biomass, was being converted into ${ }^{14} \mathrm{CO}_{2}$ and removed in the evaporation step.

\section{Potential photochemical efficiency of PSII}

Algae exposed to PtNPs at 2 and $80 \mathrm{mg}$ Pt/L, along with a control, were incubated for $48 \mathrm{~h}$ in triplicate $100 \mathrm{~mL}$ flasks of $25 \mathrm{~mL}$ suspension. After 2, 24 and $48 \mathrm{~h}$ incubation, samples of $3.5 \mathrm{~mL}$ were drawn from all replicates, and upon $1 \mathrm{~h}$ of dark acclimation, fluorescence variables were measured by Fast Repetition Rate Fluorometry (FRRF) using a FastOcean FRR plus FastAct fluorometer (Chelsea Technologies Group Ltd). Six acquisitions were run per sample and each acquisition comprised of 36 sequence repeats with saturation/relaxation phases of 100/40 flashlets per sequence and a 2/50 $\mu$ s pitch. By use of the program FastPro8 @ (Version 1.0.50, Kevin Oxborough, Chelsea Technologies Group Ltd), the potential photochemical efficiency of PSII (Fv/Fm) was obtained, reflective of changes in the photochemical energy conversion efficiency. ${ }^{3}$ Specific blank corrections with pure medium and PtNPs suspension of 80 $\mathrm{mg} \mathrm{Pt} / \mathrm{L}$ in medium were carried out to rule out a possible direct increase of the fluorescence signal due to the presence of PtNPs. Fluorescence blank readings were lower than $10 \%$ of the sample fluorescence (22\% for 2 h measurements). 


\section{References}

104 (1) ISO. Water quality - Fresh water algal growth inhibition test with unicellular green algae.

105 ISO8692:2012; International Organization for Standardization: Geneva, Switzerland, 2012.

106 (2) Harris, E. H. Chlamydomonas in the laboratory. The Chlamydomonas Sourcebook. Introduction to

107 Chlamydomonas and its laboratory use, 2nd ed.; Academic Press, San Diego, California, USA, 2009.

108 (3) Greene, R. M.; Kolber, Z. S.; Swift, D. G.; Tindale, N. W.; and Falkowski, P. G. Physiological

109 Limitation of Phytoplankton Photosynthesis in the Eastern Equatorial Pacific Determined from Variability

110 in the Quantum Yield of Fluorescence. Limnol. Oceanogr. 1994, 39, 1061-1074. 
114 Table S1. Separation program used for AsFlFFF measurements with an outflow of $1.0 \mathrm{~mL} / \mathrm{min}$.

\begin{tabular}{|lclc|}
\hline & Time [s] & & Crossflow [mL/min] \\
\hline Injection/focusing & \multirow{2}{*}{300} & $\begin{array}{l}\text { Injection flow } \\
0.2 \mathrm{~mL} / \mathrm{min}\end{array}$ & 2 \\
\hline \multirow{3}{*}{ Crossflow } & 1200 & constant & 1 \\
& 300 & linear decay & 0 \\
& 300 & constant & 0 \\
\hline
\end{tabular}

117 Table S2. Diameters and widths of the different size distributions and Pt recoveries obtained by AsFlFFF.

\begin{tabular}{|c|ccc|ccc|}
\hline & \multicolumn{3}{|c|}{ ISO 8692 medium } & \multicolumn{3}{c|}{ TAP4 medium } \\
\hline $\begin{array}{c}\text { Time for PtNPs } \\
\text { in media (h) }\end{array}$ & $\begin{array}{c}\text { Size } \\
(\mathrm{nm})\end{array}$ & $\begin{array}{c}\text { Width } \\
(\mathrm{nm})\end{array}$ & $\begin{array}{c}\text { Recovery } \\
(\%)\end{array}$ & Size (nm) & $\begin{array}{c}\text { Width } \\
(\mathrm{nm})\end{array}$ & $\begin{array}{c}\text { Recovery } \\
(\%)\end{array}$ \\
\hline 0 & 9.5 & 7.2 & $78 \pm 4$ & 9.5 & 7.1 & $80 \pm 4$ \\
1 & 9.4 & 7.1 & $82 \pm 4$ & 9.5 & 6.9 & $80 \pm 4$ \\
5 & 9.5 & 7.1 & $87 \pm 4$ & 8.4 & 6.1 & $75 \pm 4$ \\
24 & 9.2 & 7.4 & $83 \pm 4$ & 8.6 & 5.4 & $62 \pm 3$ \\
48 & 9.6 & 7.6 & $77 \pm 4$ & 8.2 & 5.1 & $63 \pm 3$ \\
\hline
\end{tabular}



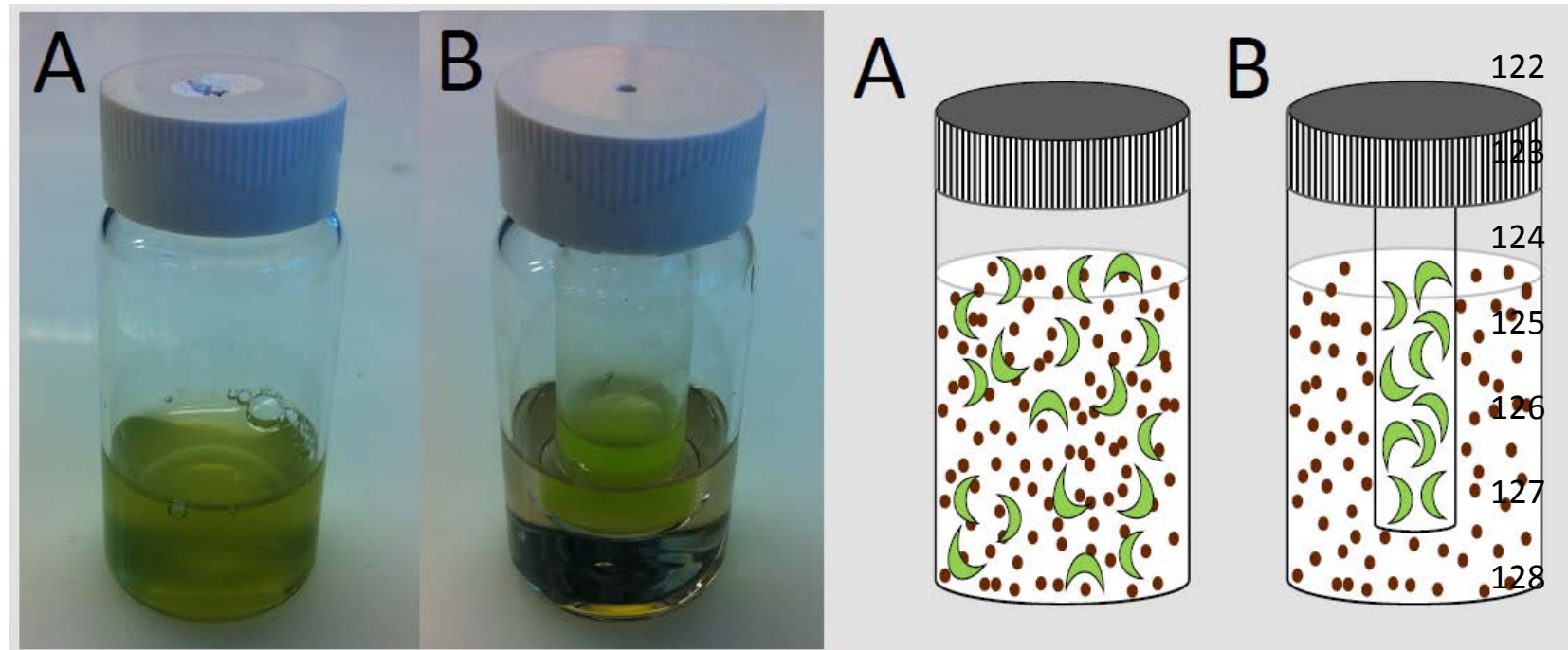

130 Figure S1. Photos and illustrations of the test setup used for determining shading effects in the algal 131 growth inhibition and ${ }^{14} \mathrm{C}$-assimilation tests. A) The setup used for standard testing and B) The double132 vial setup, allowing for separation of algal cells in the smaller inner-vial from the surrounding PtNP 133 suspension in the larger outer-vial. 
S4 Flow cytometry gating strategies
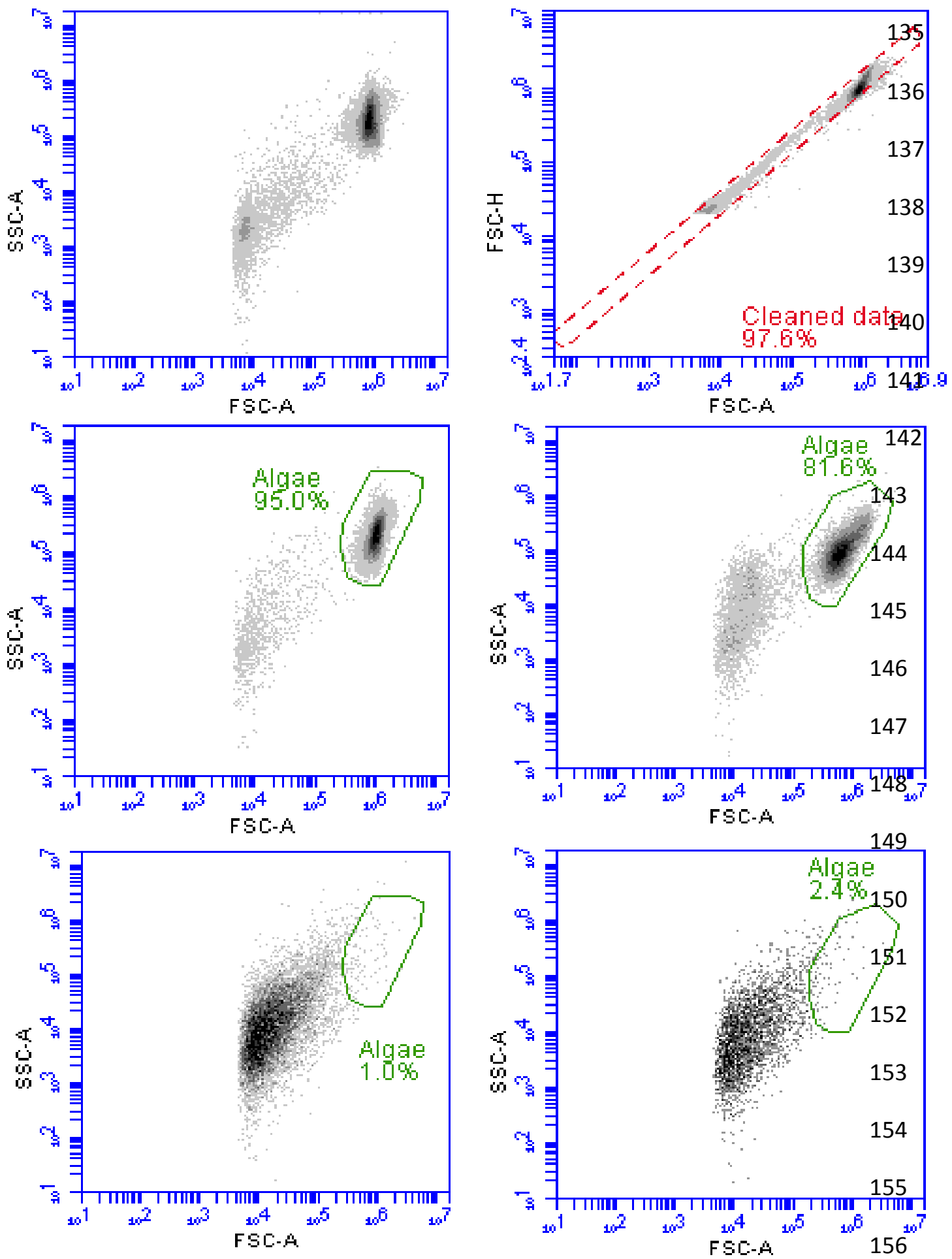

157 Figure S2. Flow cytrometry gating strategy. A) Raw data for C. reinhardtii upon 48 h exposure to PtNPs 158 (80 mg PtL), B) Removing doublets with "cleaned data” gate, C) Algal gate for unexposed C. reinhardtii, 159 D) Algal gate for unexposed P. subcapitata, E) PtNPs in TAP4 medium, and F) PtNPs in ISO medium. $160 \quad$ FSC $=$ Forward scatter, SSC $=$ Side scatter, $\mathrm{A}=$ Area, $\mathrm{H}=$ Height. 

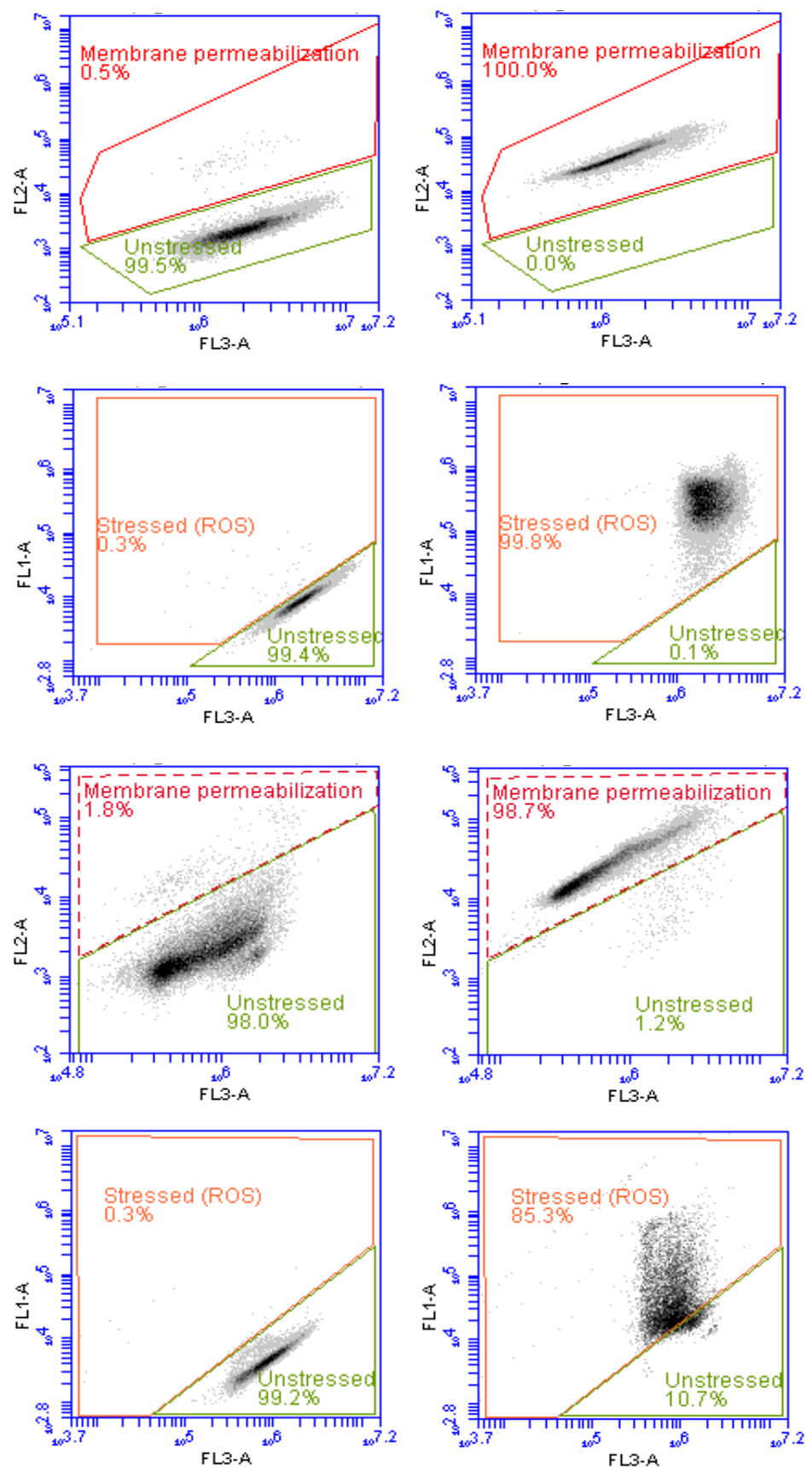

Figure S3. Flow cytrometry gating strategy for biological end points using flow cytometer BD Accuri C6. The four graphs on top apply to $C$. reinhardtii and the four below, to $P$. subcapitata. The autofluorescence is measured in the fluorescence channel 3 (FL3), membrane permeabilization with the fluorescent probe propidium iodide in fluorescense channel 2 (FL2) and oxidative stress with the fluorescent probe CellROX green in fluorescense channel 1 (FL1). The gates are determined based on the negative and positive controls, except for CellROX in $P$. subcapitata, as $\mathrm{H}_{2} \mathrm{O}_{2}$ was not a usable postitive control for this alga/medium. The experiments are considered valid, as a very clear response was obtained from algae exposed to PtNPs. 

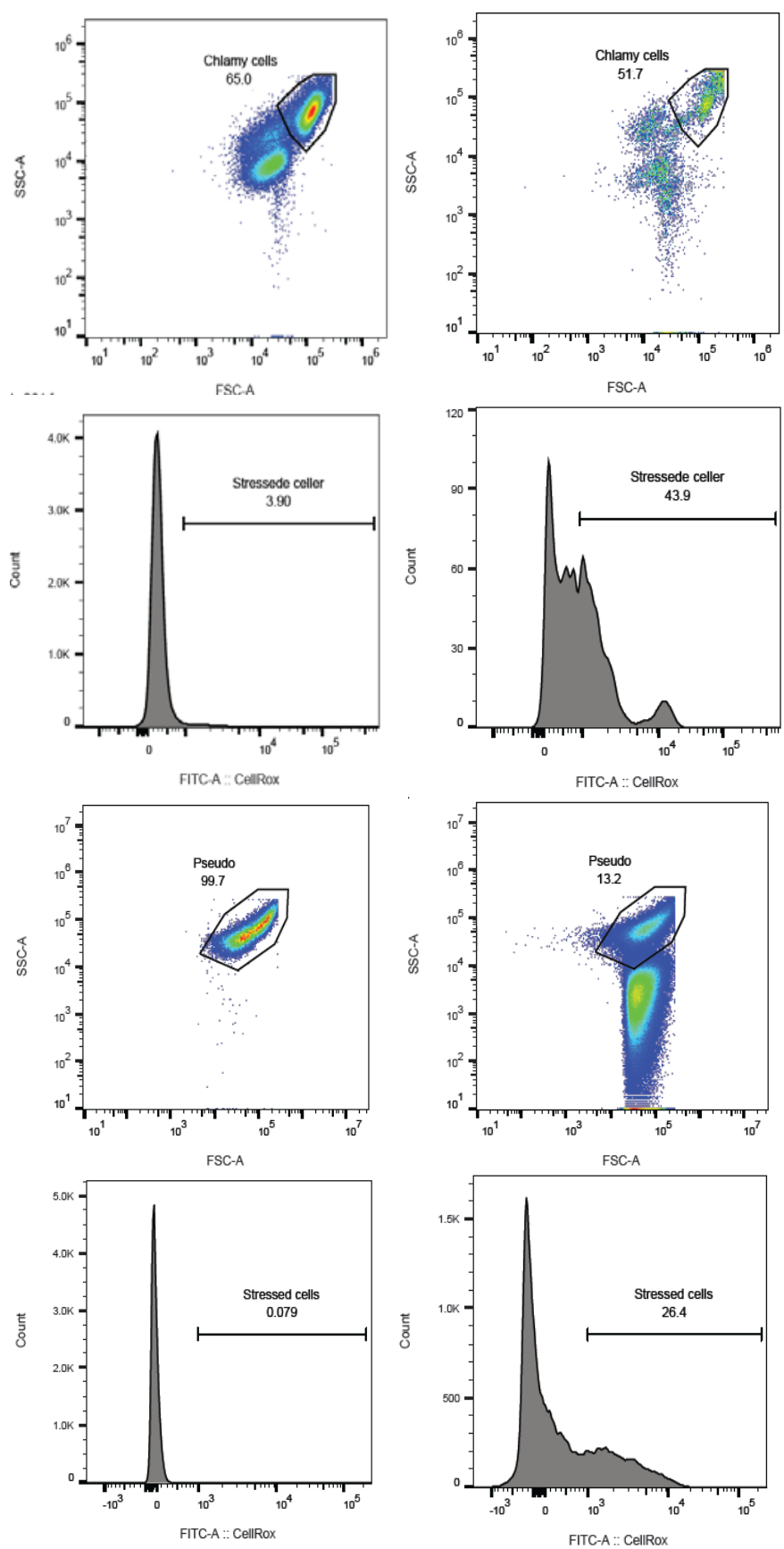

171 Figure S4. Flow cytrometry gating strategy for biological end points using flow cytometer BD

172 FACSCanto II. The four top graphs apply to $C$. reinhardtii and the four below, to $P$. subcapitata. The oxidative stress response is determined by the fluorescense of CellROX green in the FITC channel 1 of algal cells in the assigned gates. For $C$. reinhardtii a likely contamination was observed, and algal cells were distinguished by autoflourescence of algal pigments at $690 \mathrm{~nm}$. The gates are determined based on negative and positive controls, except for CellROX in $P$. subcapitata, as $\mathrm{H}_{2} \mathrm{O}_{2}$ was not a usable positive control for this alga/medium. The experiments are considered valid, as a very clear response was obtained from algae exposed to PtNPs. 
PtNPs TAP4

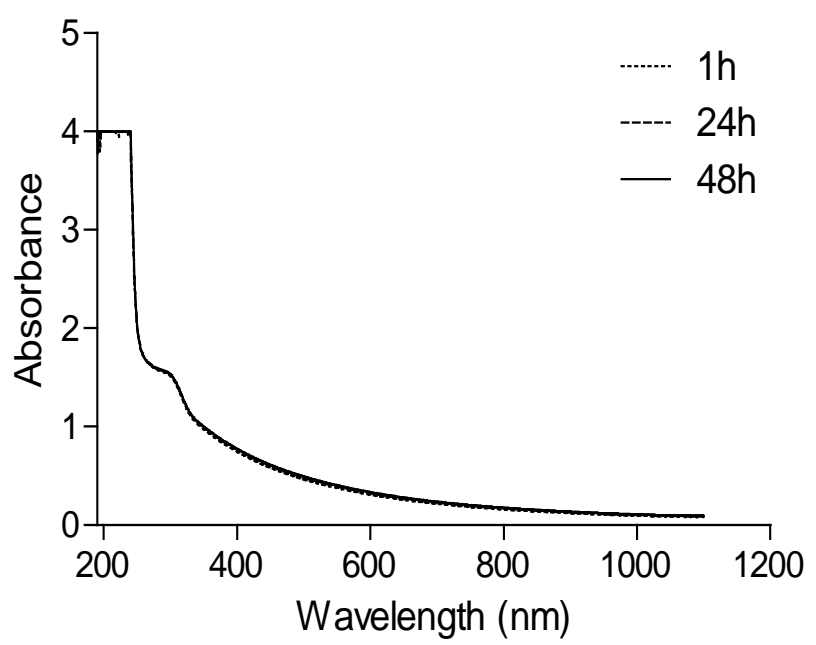

PtNPs ISO

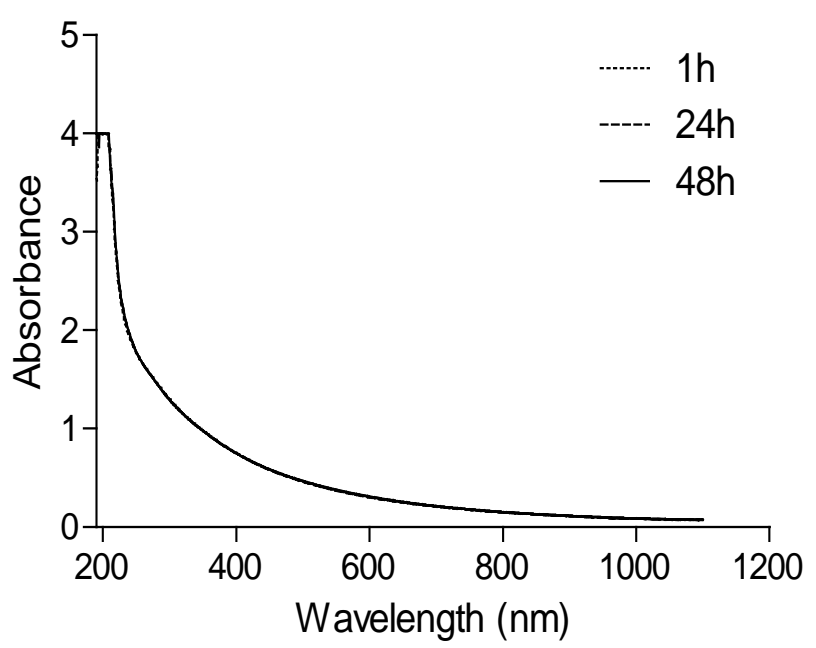

Figure S5. Absorbance spectra for PtNPs (80 mg Pt/L) suspended in ISO and TAP4 algal media after 1, 24, and $48 \mathrm{~h}$. 
ISO
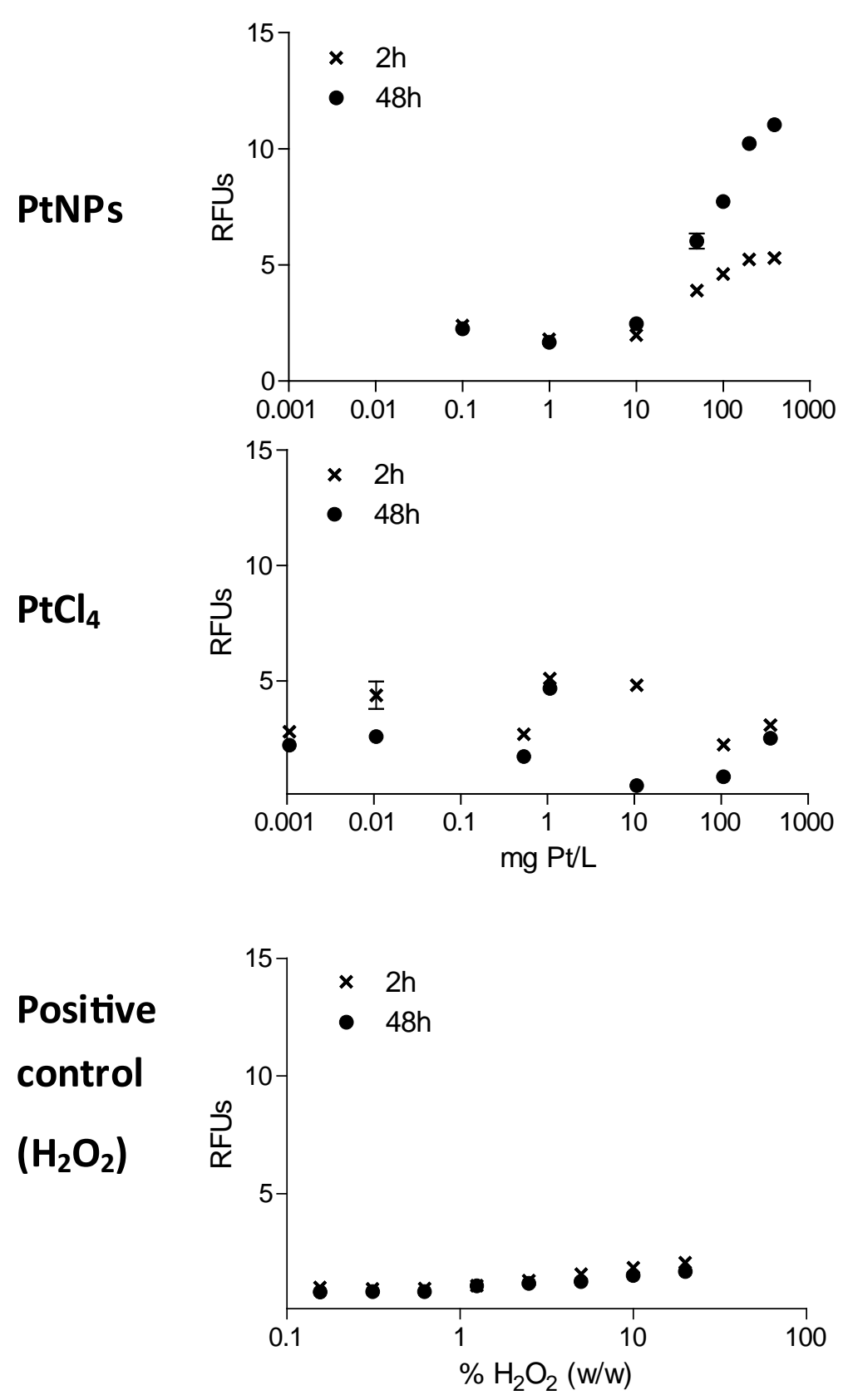

TAP4
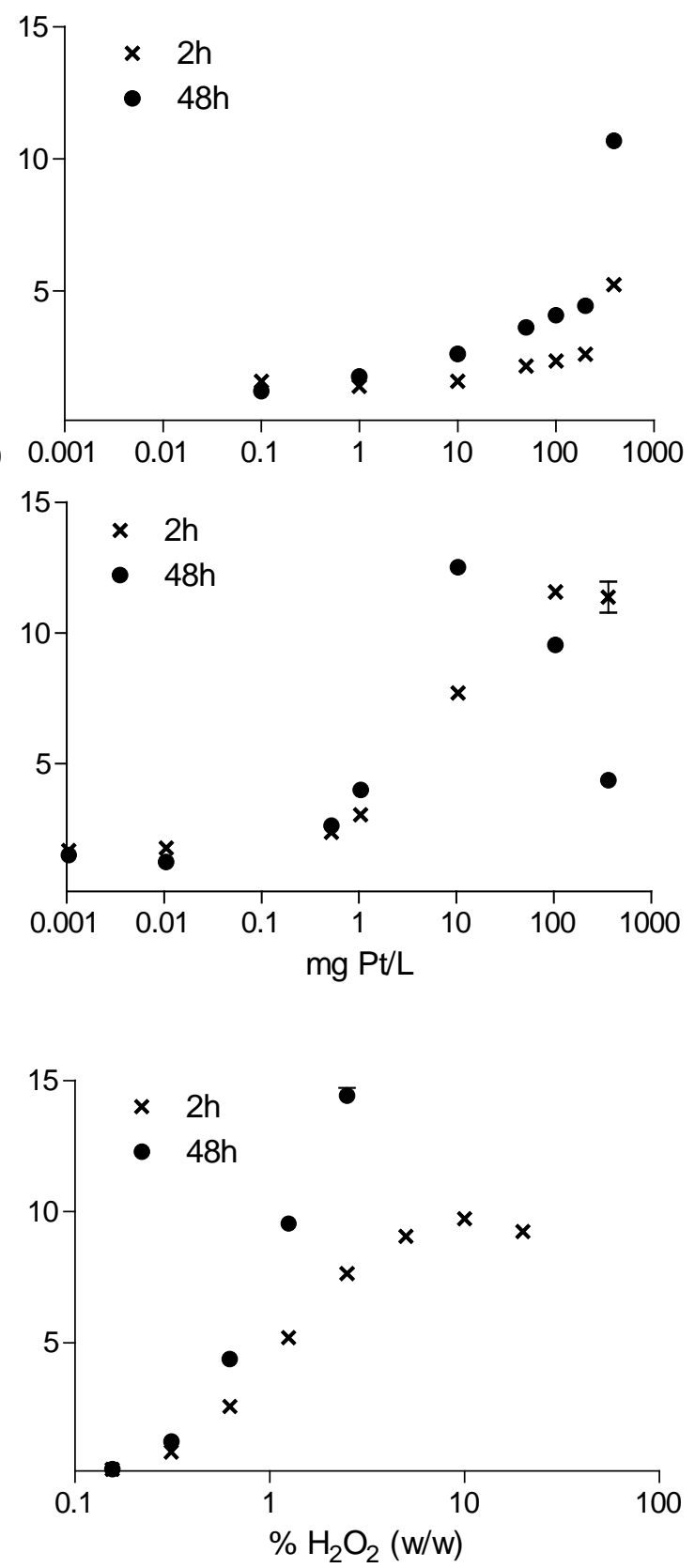

Figure S6. Abiotic ROS generation of PtNPs, $\mathrm{PtCl}_{4}$ and the positive control $\left(\mathrm{H}_{2} \mathrm{O}_{2}\right)$ upon 2 and $48 \mathrm{~h}$ suspension in ISO and TAP4 algal media, given as relative fluorescence units (RFUs) determined by the fluorescence of DCF from the tested suspension, relative to the fluorescence of the background (DCF in the respective media). The error bars represent standard deviations $(n=3)$. The fluorescence exceeded the 189 detection range in the positive controls of 5-20\%w/w $\mathrm{H}_{2} \mathrm{O}_{2}$ in TAP4 medium for $48 \mathrm{~h}$ measurements. 


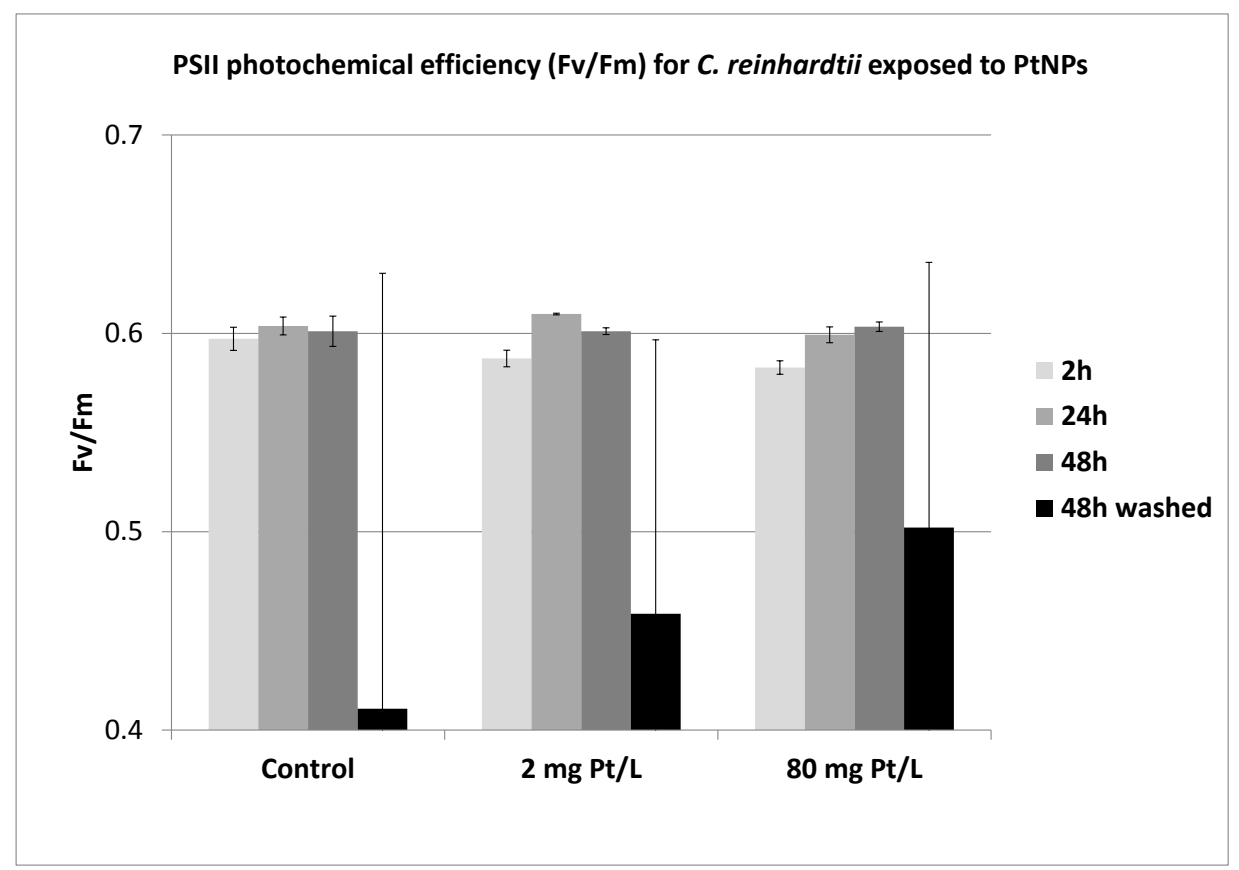

PSII photochemical efficiency (Fv/Fm) P. subcapitata exposed to PtNPs

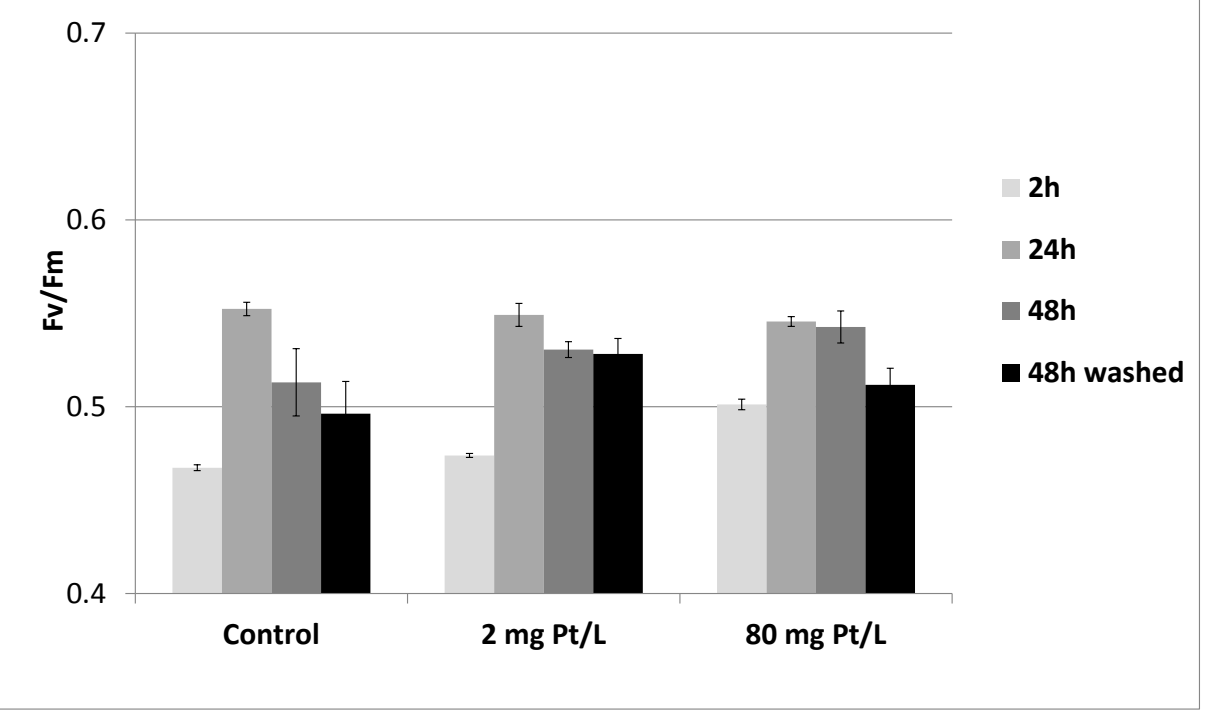

Figure S7. Fast Repetition Rate Fluorometry (FRRF) measurements of the potential PSII photochemical efficiency (Fv/Fm) for C. reinhardtii and P. subcapitata upon 2, 24 and 48 h exposure to 0, 2 or $80 \mathrm{mg}$ $\mathrm{Pt} / \mathrm{L}$. Measurements were conducted for the algae in the PtNP suspensions, and for $48 \mathrm{~h}$ measurements also for algal cells washed with medium through a filter (48 $\mathrm{h}$ washed). The error bars represent standard 198 deviations $(\mathrm{n}=3)$. 

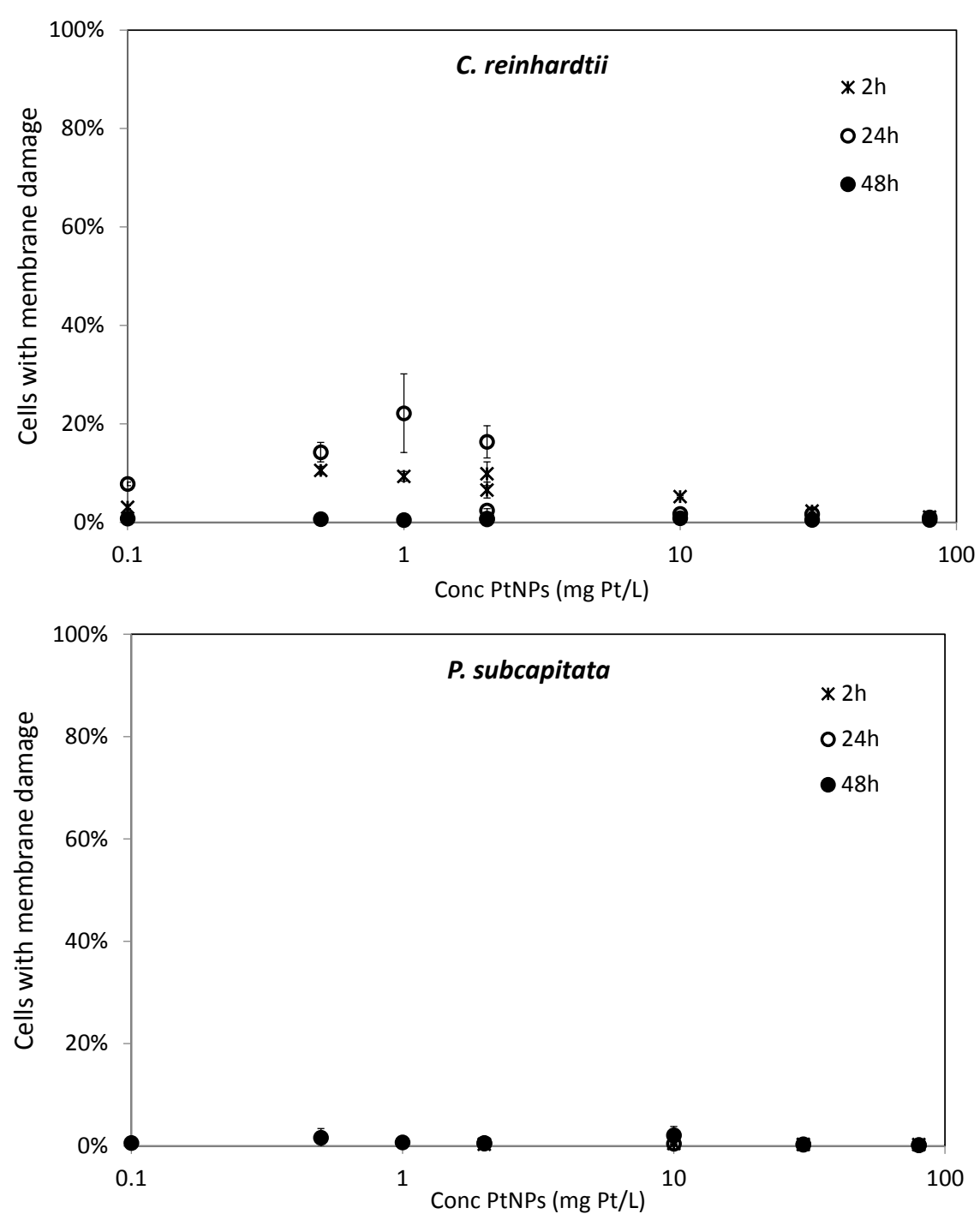

201 Figure S8. Membrane damage in C. reinhardtii and P. subcapitata upon 2, 24 and 48 h exposure to PtNPs 202 in two parallel tests with low concentrations (0.1; 0.5; 1 and $2 \mathrm{mg}$ Pt/L nominal) and high concentrations 203 (2, 10, 30 and $80 \mathrm{mg} \mathrm{Pt} / \mathrm{L})$, respectively. The error bars represent standard deviations (n=3). 


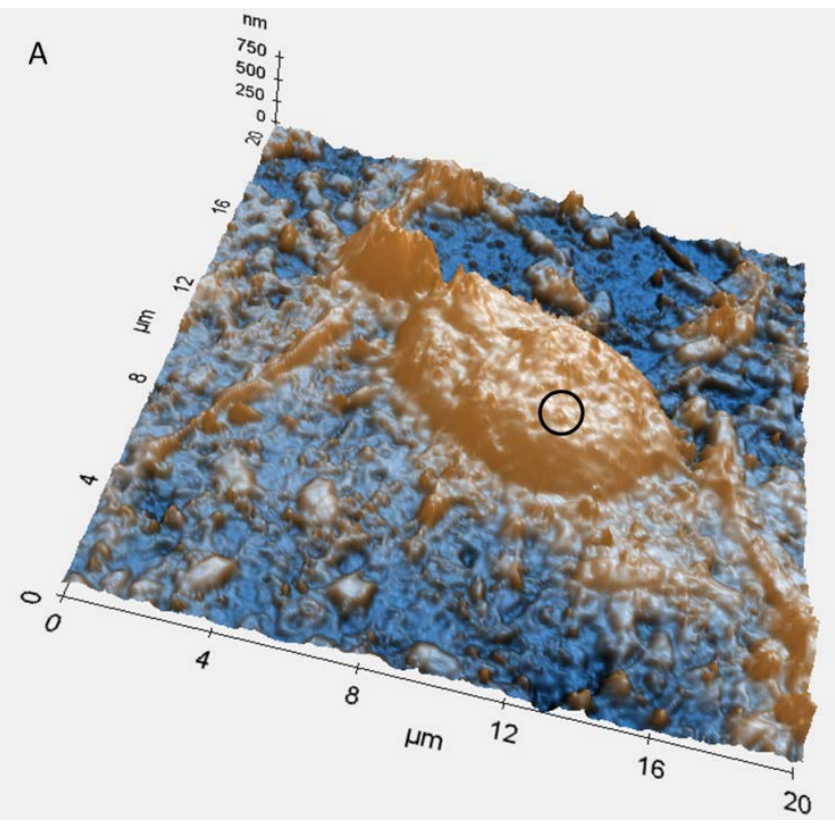

\section{B}

Figure S9. AFM visualizations of algal cells after $48 \mathrm{~h}$ incubation with PtNPs showing a single cell of $C$. reinhardtii (A) and two cells of $P$. subcapitata (B). The circle shown on the image of $C$. reinhardtii (A) shows the likely presence of PtNP agglomerates. According to the line scan completed for $P$. subcapitata (B), structures of app. $100 \mathrm{~nm}$ are identified on the cell surface, which may likely be PtNPs agglomerates. 